\title{
ROTATIONAL UNIQUENESS CONDITIONS UNDER OBLIQUE FACTOR CORRELATION METRIC
}

\author{
CAREL F.W. PEETERS
}

\begin{abstract}
In an addendum to his seminal 1969 article Jöreskog stated two sets of conditions for rotational identification of the oblique factor solution under utilization of fixed zero elements in the factor loadings matrix [12]. These condition sets, formulated under factor correlation and factor covariance metrics, respectively, were claimed to be equivalent and to lead to global rotational uniqueness of the factor solution. It is shown here that the conditions for the oblique factor correlation structure need to be amended for global rotational uniqueness, and hence, that the condition sets are not equivalent in terms of unicity of the solution.
\end{abstract}

Key words: Factor analysis; Oblique rotation; Rotational uniqueness; Unrestricted factor model

\section{INTRODUCTION}

Suppose $\boldsymbol{\Sigma}=\boldsymbol{\Lambda} \boldsymbol{\Phi} \boldsymbol{\Lambda}^{\mathrm{T}}+\boldsymbol{\Psi}$ is the usual oblique factor analysis model. Here, $\boldsymbol{\Lambda} \in \mathbb{R}^{p \times m}$ is a matrix of factor loadings in which each element $\lambda_{j k}$ is the loading of the $j$ th variable on the $k$ th factor, $j=1, \ldots, p, k=1, \ldots, m ; \boldsymbol{\Phi} \in \mathbb{R}^{m \times m}$ denotes the factor covariance matrix; and $\boldsymbol{\Psi} \in \mathbb{R}^{p \times p} \equiv \operatorname{diag}\left[\psi_{11}, \ldots, \psi_{p p}\right]$ contains the error variances. In the remainder we will assume the usual regularity assumptions: (a) $\operatorname{rank}(\boldsymbol{\Lambda})=m$; (b) $\psi_{j j}>0 \forall j$; and (c) $(p-m)^{2}-p-m \geqslant 0$, simply stating nonnegative degrees of freedom for existence.

As is well-known, the factor model is inherently underidentified, implying that $\boldsymbol{\Sigma}$ does not have a unique solution without imposing restrictions. Given $\boldsymbol{\Psi}$, two factor models defined by $\{\boldsymbol{\Lambda}, \boldsymbol{\Phi}\}$ and $\left\{\boldsymbol{\Lambda}^{\ddagger}, \boldsymbol{\Phi}^{\ddagger}\right\}$ are equivalent if there exists a mapping $\delta$ : $\{\boldsymbol{\Lambda}, \boldsymbol{\Phi}\} \longrightarrow\left\{\boldsymbol{\Lambda}^{\ddagger}, \boldsymbol{\Phi}^{\ddagger}\right\}$ such that $\boldsymbol{\Sigma}(\boldsymbol{\Lambda}, \boldsymbol{\Phi}, \boldsymbol{\Psi})=\boldsymbol{\Sigma}[\delta(\boldsymbol{\Lambda}, \boldsymbol{\Phi}), \boldsymbol{\Psi}]$. For the factor model we find $\boldsymbol{\Sigma}=(\boldsymbol{\Lambda} \mathbf{R})\left[\mathbf{R}^{-1} \boldsymbol{\Phi}\left(\mathbf{R}^{\mathrm{T}}\right)^{-1}\right](\boldsymbol{\Lambda} \mathbf{R})^{\mathrm{T}}+\boldsymbol{\Psi}$, where $\mathbf{R} \in \mathbb{R}^{m \times m}$ is an arbitrary nonsingular matrix, implying that there is an infinite number of alternative matrices $\boldsymbol{\Lambda}^{\ddagger}=\boldsymbol{\Lambda} \mathbf{R}$ and $\boldsymbol{\Phi}^{\ddagger}=\mathbf{R}^{-1} \boldsymbol{\Phi}\left(\mathbf{R}^{\mathrm{T}}\right)^{-1}$ that generate the same covariance structure $\boldsymbol{\Sigma}$. The operation $\boldsymbol{\Lambda} \mapsto \boldsymbol{\Lambda} \mathbf{R}$ is termed 'rotation', and $\boldsymbol{\Lambda}$ and $\boldsymbol{\Phi}$ are said to be globally rotationally unique iff $\mathbf{R}=\mathbf{I}_{m}$.

Finding conditions to ensure (global) rotational uniqueness has been an active area of research and debate in the factor analysis community. Building on Howe [8], Jöreskog 11] conjectured sufficient conditions for uniqueness for both the orthogonal $\left(\boldsymbol{\Phi}=\mathbf{I}_{m}\right)$ and oblique factor model through specification of fixed elements in $\boldsymbol{\Lambda}$ and $\boldsymbol{\Phi}$. Dunn [6] showed, especially for the orthogonal model, that Jöreskog's conditions were not sufficient. His substitute conditions based on fixed zero elements are sufficient for local rotational uniqueness only, in the sense that $2^{m}$ combinations of polarity reversals in the columns of $\boldsymbol{\Lambda}$ are allowed, giving that $\mathbf{R}$ is then $\operatorname{diag}[ \pm 1, \ldots, \pm 1]$. Jennrich [10] gave sufficient conditions for local rotational uniqueness under reflections for the orthogonal model when the fixed elements are 
arbitrary. These works inspired Jöreskog to write an addendum to his 1969 article [12], focussing on reformulating the sufficiency conditions for the oblique factor solution with fixed zero elements. He gave the following conditions:

(C1) Let $\boldsymbol{\Lambda}$ have at least $m-1$ fixed zeroes in each column;

(C2) Let $\operatorname{rank}\left(\boldsymbol{\Lambda}^{[k]}\right)=m-1$, where $\boldsymbol{\Lambda}^{[k]}, k=1, \ldots, m$, is the submatrix of $\boldsymbol{\Lambda}$, consisting of the rows of $\boldsymbol{\Lambda}$ which have fixed zero elements in the $k$ th column with these zeroes deleted;

(C3) Let $\boldsymbol{\Phi}$ be a symmetric positive definite matrix with $\operatorname{diag}(\boldsymbol{\Phi})=\mathbf{I}_{m}$ (i.e., $\boldsymbol{\Phi}$ is a correlation matrix);

and conjectured that $\mathrm{C} 1-\mathrm{C} 3$ are sufficient for obtaining global rotational uniqueness. Moreover, he conjectured that conditions $\mathrm{C} 1$ and $\mathrm{C} 3$ are equivalent to:

$\left(\mathrm{C}^{*}\right)$ Let $\boldsymbol{\Lambda}$ have at least $m-1$ fixed zeroes in each column and one fixed non-zero value in each column, the latter values being in different rows;

and subsequently proved global rotational uniqueness under pairing of conditions $\mathrm{C} 2$ and $\mathrm{C}^{*}$.

Many recent texts follow Jöreskog [12] in stating that conditions C1-C3 are sufficient for (rotational) uniqueness [e.g., 9, 2]. However, it will be shown that conditions $\mathrm{C} 1-\mathrm{C} 3$ are not sufficient for global rotational uniqueness but local rotational uniqueness only and, hence, that conditions $\mathrm{C} 1$ and $\mathrm{C} 3$ are not equivalent to $\mathrm{C}^{*}$ in terms of unicity of the solution. Although this result may be implicitly known or be considered tacit knowledge, here it is made explicit.

In the remainder, condition set C1-C3 will be amended with an additional condition. It will then be shown that the amended condition set is sufficient for obtaining global rotational uniqueness, implying that $\mathrm{C} 1-\mathrm{C} 3$ do not lead to global uniqueness and the non-equivalence of conditions $\mathrm{C} 1$ and $\mathrm{C} 3$ to $\mathrm{C}^{*}$. Section 3 concludes with a discussion.

\section{Global Rotational Uniqueness Under diag $(\boldsymbol{\Phi})=\mathbf{I}_{m}$}

Consider the following addition to conditions C1-C3:

(C4) Let in each column of $\boldsymbol{\Lambda}$ one parameter non-fixed by condition C1 be polarity truncated to take only positive or negative values, that is: In each column of $\boldsymbol{\Lambda}$ one element is to adopt either strict positivity $\left(\lambda_{j k}>0\right)$, or strict negativity $\left(-\lambda_{j k}>0\right)$.

Proposition 1. Let the mapping $\delta:\{\boldsymbol{\Lambda}, \boldsymbol{\Phi}\} \longrightarrow\left\{\boldsymbol{\Lambda}^{\ddagger}, \boldsymbol{\Phi}^{\ddagger}\right\}$ be defined by $\boldsymbol{\Lambda}^{\ddagger}=$ $\Lambda \mathbf{R}$ and $\boldsymbol{\Phi}^{\ddagger}=\mathbf{R}^{-1} \boldsymbol{\Phi}\left(\mathbf{R}^{\mathrm{T}}\right)^{-1}$, where $\mathbf{R} \in \mathbb{R}^{m \times m}$ denotes an arbitrary nonsingular matrix. If conditions $\mathrm{C} 1-\mathrm{C} 4$ hold, then $\mathbf{R}=\mathbf{I}_{m}$.

Proof. Let conditions $\mathrm{C} 1-\mathrm{C} 4$ hold on $\{\boldsymbol{\Lambda}, \boldsymbol{\Phi}\}$. We start by showing that $\mathbf{R}$ is diagonal under conditions $\mathrm{C} 1-\mathrm{C} 2$, which can be shown with an argument analogous to Anderson [1, pp. 576-577]. Under given conditions it is always possible to find a permutation matrix $\mathbf{P}_{1}$ of respective dimension $p \times p$, such that $\mathbf{P}_{1} \boldsymbol{\Lambda}$ gives a block lower right triangular form on $\boldsymbol{\Lambda}^{[1]}$. We then have

$$
\boldsymbol{\Lambda}^{\prime}=\mathbf{P}_{1} \boldsymbol{\Lambda}=\left[\begin{array}{cc}
\mathbf{0} & \boldsymbol{\Lambda}^{[1]} \\
\boldsymbol{\lambda}_{(1)} & \boldsymbol{\Lambda}_{[1]}
\end{array}\right]
$$


where $\mathbf{0}$ is a $(m-1)$-dimensional null vector, $\boldsymbol{\lambda}_{(1)} \in \mathbb{R}^{(p-m+1) \times 1}, \boldsymbol{\Lambda}^{[1]} \in$ $\mathbb{R}^{(m-1) \times(m-1)}$, and $\boldsymbol{\Lambda}_{[1]} \in \mathbb{R}^{(p-m+1) \times(m-1)}$. Now, let

$$
\mathbf{R}=\left[\begin{array}{ll}
r_{11} & \mathbf{r}_{12} \\
\mathbf{r}_{21} & \mathbf{R}_{22}
\end{array}\right]
$$

where $r_{11}$ is a scalar, $\mathbf{r}_{12} \in \mathbb{R}^{1 \times(m-1)}, \mathbf{r}_{21} \in \mathbb{R}^{(m-1) \times 1}$, and $\mathbf{R}_{22} \in \mathbb{R}^{(m-1) \times(m-1)}$. Then

$$
\left(\boldsymbol{\Lambda}^{\prime}\right)^{\ddagger}=\boldsymbol{\Lambda}^{\prime} \mathbf{R}=\left[\begin{array}{cc}
\boldsymbol{\Lambda}^{[1]} \mathbf{r}_{21} & \boldsymbol{\Lambda}^{[1]} \mathbf{R}_{22} \\
r_{11} \boldsymbol{\lambda}_{(1)}+\boldsymbol{\Lambda}_{[1]} \mathbf{r}_{21} & \boldsymbol{\lambda}_{(1)} \mathbf{r}_{12}+\boldsymbol{\Lambda}_{[1]} \mathbf{R}_{22}
\end{array}\right] .
$$

As the rank of $\boldsymbol{\Lambda}^{[1]}$ is $m-1, \mathbf{r}_{21}$ should be a null vector. We may follow the same procedure for each respective remaining submatrix $\boldsymbol{\Lambda}^{[k]}$. That is, we can find an $(m \times m)$-dimensional permutation matrix $\mathbf{P}_{2}$ such that $\mathbf{P}_{1}^{\prime} \mathbf{\Lambda} \mathbf{P}_{2}$ has a structure as in (11) with the index 1 replaced by $k$ (we may always find a pair of permutation matrices $\mathbf{P}_{1}^{\prime}$ and $\mathbf{P}_{2}$, such that $\mathbf{P}_{1}^{\prime} \mathbf{\Lambda} \mathbf{P}_{2}$ gives a block lower right triangular form on $\left.\boldsymbol{\Lambda}^{[k]}\right)$. From the accompanying reorderings $\mathbf{P}_{2}^{\mathrm{T}} \mathbf{R} \mathbf{P}_{2}$ implied by the identity

$$
\begin{aligned}
& \mathbf{P}_{1}^{\prime} \boldsymbol{\Sigma}\left(\mathbf{P}_{1}^{\prime}\right)^{\mathrm{T}} \\
& =\left(\mathbf{P}_{1}^{\prime} \boldsymbol{\Lambda} \mathbf{R}\right)\left[\mathbf{R}^{-1} \boldsymbol{\Phi}\left(\mathbf{R}^{\mathrm{T}}\right)^{-1}\right]\left(\mathbf{P}_{1}^{\prime} \boldsymbol{\Lambda} \mathbf{R}\right)^{\mathrm{T}}+\mathbf{P}_{1}^{\prime} \mathbf{\Psi}\left(\mathbf{P}_{1}^{\prime}\right)^{\mathrm{T}} \\
& =\left(\mathbf{P}_{1}^{\prime} \boldsymbol{\Lambda} \mathbf{P}_{2} \mathbf{P}_{2}^{\mathrm{T}} \mathbf{R} \mathbf{P}_{2}\right)\left[\mathbf{P}_{2}^{\mathrm{T}} \mathbf{R}^{-1} \mathbf{P}_{2} \mathbf{P}_{2}^{\mathrm{T}} \boldsymbol{\Phi} \mathbf{P}_{2} \mathbf{P}_{2}^{\mathrm{T}}\left(\mathbf{R}^{\mathrm{T}}\right)^{-1} \mathbf{P}_{2}\right]\left(\mathbf{P}_{1}^{\prime} \boldsymbol{\Lambda} \mathbf{P}_{2} \mathbf{P}_{2}^{\mathrm{T}} \mathbf{R} \mathbf{P}_{2}\right)^{\mathrm{T}}+\mathbf{P}_{1}^{\prime} \mathbf{\Psi}\left(\mathbf{P}_{1}^{\prime}\right)^{\mathrm{T}},
\end{aligned}
$$

it follows that under conditions $\mathrm{C} 1-\mathrm{C} 2, \mathbf{R}=\operatorname{diag}\left[r_{11}, \ldots, r_{m m}\right]$.

The properties of diagonal matrices are such that now $\mathbf{R}^{\mathrm{T}}=\mathbf{R}$ and $\mathbf{R}^{-1}=$ $\operatorname{diag}\left[r_{11}^{-1}, \ldots, r_{m m}^{-1}\right]$. Superimposing condition C3 then implies

$$
\phi_{k k}^{\ddagger}=r_{k k}^{-2} \phi_{k k}=r_{k k}^{-2}=1 \quad \forall k,
$$

giving that $r_{k k}= \pm 1$, and $\mathbf{R}=\operatorname{diag}[ \pm 1, \ldots, \pm 1]$.

By demanding that each column of $\boldsymbol{\Lambda}$ has a polarity truncation, column multiplication by -1 is no longer possible as this would imply a reversal of the polarity truncation (direction inequality symbol). Superimposing condition C4 on C1-C3 thus gives that $\mathbf{R}=\mathbf{I}_{m}$. The proposition follows.

Remark 1. The proof of Proposition 1 implies, contrary to previous conjectures, that conditions C1-C3 are not sufficient for global rotational uniqueness as they provide local rotational uniqueness only and, hence, that conditions $\mathrm{C} 1$ and $\mathrm{C} 3$ are not equivalent to $\mathrm{C}^{*}$ as the pairing of $\mathrm{C} 2-\mathrm{C}^{*}$ does provide global rotational uniqueness [12]. Moreover, the proposition indicates how inequality restrictions can aid in the attainment of global rotational uniqueness.

Remark 2. Instead of using strict positivity or strict negativity truncations as formulated in condition $\mathrm{C} 4$ we could also use strict polarity truncations by (arbitrary) constants, that is: Every column of $\boldsymbol{\Lambda}$ should contain either $\lambda_{j k}>c_{k} \in \mathbb{R}^{+}$or $-\lambda_{j k}>c_{k} \in \mathbb{R}^{+}$. While this will produce global rotational uniqueness whence superimposed on $\mathrm{C} 1-\mathrm{C} 3$ along the same lines as $\mathrm{C} 4$, it may not be practical in a research setting. It may, for example, be possible to specify $\lambda_{j k}>c_{k} \in \mathbb{R}^{+}$while the true parameter value $0 \leqslant \lambda_{j k}<c_{k}$ (in the positive reflection). One would then run into estimation trouble in numerical applications. A related issue lies in choosing the loading elements for column polarity fixation. For (Bayesian) estimation efficiency (see Discussion section) condition C4 should be imposed on loadings that, from prior knowledge or theory, are believed to be large. 
Remark 3. Please note that rotational uniqueness of $\boldsymbol{\Lambda}$ will not guarantee identifiability of the FA model [4], as underidentification of $\boldsymbol{\Psi}$ may imply underidentification of $\boldsymbol{\Lambda}$. However, if the regularity assumptions stated in the introduction hold, then conditions C1-C4 will, next to rotational uniqueness, also provide identifiability. If unsure if identifiability is obtained, one could endeavor on algebraically checking (local) identification utilizing the Wald rank rule [3].

\section{Discussion}

The following question deserves some exploration: What are reasons to prefer condition set $\mathrm{C} 1-\mathrm{C} 4$ above the pairing $\mathrm{C} 2-\mathrm{C}^{*}$ ? Before delving into possible answers it is stated why the addition of condition $\mathrm{C} 4$ is deemed important when working in factor correlation metric.

Not attaining global rotational uniqueness in factor correlation metric under C1-C3 does not hamper maximum likelihood estimation, as any local minimum has equivalent representations through simple polarity reflections. In obtaining factor loading standard errors or Bayesian estimates the situation is a little more intricate as the parameter space under C1-C3 is multimodal. The modes (defined over polarity reflections) should be widely separated in order for unimodal normal approximations or resampling techniques to yield valid estimates of the standard errors (see, Dolan and Molenaar [e.g., 5]). As Bayesian modeling proceeds through exploration of posterior space, posterior estimates will be flawed when transition probability between modes is non-negligible. Imposing $\mathrm{C} 4$ can aid when the modes are not well-separated. In Bayesian modeling, for example, imposing C4 implies a truncation of the posterior density and will restrict posterior simulation to a single mode - a fact that has been recognized (for the orthogonal factor model) by Geweke and Zhou [7].

A first reason for preferring $\mathrm{C} 1-\mathrm{C} 4$ above the pairing $\mathrm{C} 2-\mathrm{C}^{*}$ might be found in the topic of arbitrary units of measurement. Many (psychological) tests have units of measurement with no intrinsic meaning. Let $\mathbf{D}$ be a diagonal matrix with positive diagonal elements that indicate a change in test score units. Then $\mathbf{D} \boldsymbol{\Sigma} \mathbf{D} \equiv \boldsymbol{\Sigma}^{\dagger}$. If $\boldsymbol{\Lambda}$ is identified by $\mathrm{C} 1-\mathrm{C} 4$, then $\mathbf{D} \boldsymbol{\Lambda} \equiv \boldsymbol{\Lambda}^{\dagger}$ is similarly identified. If $\boldsymbol{\Lambda}$ is identified by the pairing $\mathrm{C} 2-\mathrm{C}^{*}$, then each column of $\mathbf{D} \boldsymbol{\Lambda}$ has to be renormalized [1, p. 557].

A second reason for preference may be that the condition set C1-C4 is less restrictive on $\boldsymbol{\Lambda}$. Consider the unrestricted factor model. Unrestricted solutions correspond to exploratory factor analysis (EFA) in the sense that only minimal restrictions are placed on the model to achieve at least a local rotationally unique solution for $m$ factors. As such, an unrestricted solution for $m$ common factors does not restrict the factor space and will yield an optimal fit for any model with $m$ factors [13, Section 15.4]. In effect, a minimal set of restrictions based on C2 and $\mathrm{C}^{*}$ or $\mathrm{C} 1-\mathrm{C} 4$ entails a choice of rotation of the EFA model. The pairing $\mathrm{C} 2-\mathrm{C}^{*}$ imposes the minimum of $\mathrm{m}^{2}$ restrictions, equalling the number of non-redundant elements in $\mathbf{R}$, on the parameter space of $\boldsymbol{\Lambda}$. The condition set C1-C4 imposes only $m(m-1)$ fixed-value restrictions on $\boldsymbol{\Lambda}$. The parameters involved in the polarity truncations are free to be estimated in either the positive or negative range (note also that the polarity truncations do not restrict the factor space). The fewer number of restrictions on $\boldsymbol{\Lambda}$ under $\mathrm{C} 1-\mathrm{C} 4$ make it a more flexible set for unrestricted formulations of the (confirmatory) factor model. 


\section{ACKNOWLEDGEMENTS}

This research was supported by grant NWO-VICI-453-05-002 of the Netherlands Organization for Scientific Research (NWO). It was written while the author was a Ph.D. candidate at the Department of Methodology and Statistics, Utrecht University, Utrecht, the Netherlands and is part of the authors' Ph.D. thesis. This version is a postprint of: Peeters, C.F.W. (2012). Rotational Uniqueness Conditions under Oblique Factor Correlation Metric. Psychometrika, 77: 288-292.

\section{REFERENCES}

[1] T. W. Anderson. An Introduction to Multivariate Statistical Analysis. New York: Wiley, 1984.

[2] T. Asparouhov and B. Muthén. Exploratory structural equation modeling. Structural Equation Modeling, 16:397-438, 2009.

[3] P. A. Bekker, A. Merckens, and T. J. Wansbeek. Identification, Equivalent Models, and Computer Algebra. Boston: Academic Press, 1994.

[4] K. A. Bollen and K. G. Jöreskog. Uniqueness does not imply identification: A note on confirmatory factor analysis. Sociological Methods $\&$ Research, 14: $155-163,1985$.

[5] C. V. Dolan and P. C. M. Molenaar. A comparison of four methods of calculating standard errors of maximum-likelihood estimates in the analysis of covariance structure. British Journal of Mathematical \&5 Statistical Psychology, 44:359-368, 1991.

[6] J. E. Dunn. A note on a sufficiency condition for uniqueness of a restricted factor matrix. Psychometrika, 38:141-143, 1973.

[7] J. F. Geweke and G. Zhou. Measuring the pricing error of the arbitrage pricing theory. Review of Financial Studies, 9:557-587, 1996.

[8] W. G. Howe. Some contributions to factor analysis. Technical Report ORNL1919, Oak Ridge, Tennessee: Oak Ridge National Laboratory, 1955.

[9] R. H. Hoyle and J. L. Duvall. Determining the number of factors in exploratory and confirmatory factor analysis. In D. Kaplan, editor, The SAGE Handbook of Quantitative Methodology for the Social Sciences, pages 301-315. Thousand Oaks, CA: SAGE, 2004.

[10] R. I. Jennrich. Rotational equivalence of factor loading matrices with specified values. Psychometrika, 43:421-426, 1978.

[11] K. G. Jöreskog. A general approach to confirmatory maximum likelihood factor analysis. Psychometrika, 34:183-202, 1969.

[12] K. G. Jöreskog. Author's addendum. In Jay Magidson, editor, Advances in Factor Analysis and Structural Equation Models, pages 40-43. Cambridge, Massachusetts: Abt Books, 1979.

[13] S. A. Mulaik. Foundations of Factor Analysis. Boca Raton: Chapman \& Hall/CRC, 2nd edition, 2010.

(Carel F.W. Peeters) Dept. of Epidemiology \& Biostatistics, Amsterdam Public Health research institute, VU University medical center Amsterdam, Amsterdam, The NetherLANDS

E-mail address: cf.peeters@vumc.nl 